\title{
Neptune's zonal winds from near-IR Keck adaptive optics imaging in August 2001
}

\author{
Shuleen Chau Martin · Imke de Pater · Philip Marcus
}

Received: 18 August 2011 / Accepted: 20 August 2011 / Published online: 14 September 2011

(C) The Author(s) 2011. This article is published with open access at Springerlink.com

\begin{abstract}
We present H-band (1.4-1.8 $\mu \mathrm{m})$ images of Neptune with a spatial resolution of $\sim 0.06^{\prime \prime}$, taken with the W.M. Keck II telescope using the slit-viewing camera (SCAM) of the NIRSPEC instrument backed with Adaptive Optics. Images with 60-second integration times span 4 hours each on UT 20 and 21 August, 2001 and $\sim 1$ hour on UT 1 September, 2001. These images were used to characterize the overall brightness distribution on Neptune, and to determine rotations periods (which translate into wind speeds) of individual cloud features.

The images show that the spatial brightness distribution of cloud features, in particular the bright bands at midsouthern latitudes and near $30^{\circ} \mathrm{N}$, changed considerably between 1989 (Voyager era) and 2001. The brightest features extend latitudinally over several degrees, and despite the different velocities in different latitude bands, these bright features remain coherent. We show that these features are bright in part because of the foreshortening effect near the limb, which suggests that the features may be composed of small bright clouds that happen to line up near the limb.

At certain latitudes (mid-southern and northern latitudes), there is considerable dispersion in relative rotation periods (and hence zonal velocities) of faint and moderately
\end{abstract}

S.C. Martin · I. de Pater $(\bowtie)$

Department of Astronomy, 601 Campbell Hall, University

of California, Berkeley, CA 94720, USA

e-mail: imke@berkeley.edu

\section{I. de Pater}

Delft Institute of Earth Observation and Space Systems, Delft

University of Technology, 2629 HS Delft, The Netherlands

\section{P. Marcus}

Department of Mechanical Engineering, University of California, Berkeley, CA 94720, USA bright features, while there is essentially no velocity dispersion of features at $50^{\circ} \mathrm{S}$. While the zonal speeds of the brightest features are consistent with the Voyager-derived zonal-mean wind profile, there are many cloud features that do not appear to move with the flow. The data are further suggestive of oscillations in longitude, with periods $>4 \mathrm{hrs}$. We suggest that tidal forcing by Triton could play a role in exciting the waves responsible for the velocity variations of the observed period.

Keywords Infrared · Planetary systems · Planets and satellites: Neptune · Atmospheres

\section{Introduction}

The atmospheres of the giant planets are characterized by zonal winds that have been derived by tracking cloud features in their atmospheres. The winds on Jupiter, Saturn and Uranus appear to be quite stable over time. Small variations have been detected at particular latitudes in the winds on Jupiter (see e.g., Asay-Davis et al. 2011, and references therein). Asay-Davis et al., however, point out that care must be taken to check such reported variations carefullyin particular near a latitude of $8^{\circ} \mathrm{N}$ some retrieval methods incorrectly found slower zonal winds because the eastward drift of the dark projections (associated with 5- $\mu \mathrm{m}$ hot spots) "fooled" the retrieval algorithms - and such "errors" can lead to incorrect reports on changes in the zonal wind. Both Limaye (1989) and Asay-Davis et al. (2011) further argue that the Jovian zonal flow has temporal variations on timescales of hours to years that are $\sim 10 \mathrm{~m} / \mathrm{s}$, which is of order $10 \%$ of the maximum speed. Despite these reports, it is clear that overall Jupiter's zonal wind can be considered quite stable. Reports on changes in Saturn's equatorial jet 
may or may not be explained by probing different altitudes at different times (Sanchez-Lavega et al. 2007), but besides these changes the winds on this planet also appear to be quite stable. To date, absolutely no variability has been detected in Uranus's zonal wind profiles (Sromovsky et al. 2009).

On Neptune, the "canonical" zonal wind profile is a smooth fit through wind speed measurements of large and small cloud features that were identified and tracked on images taken with the Voyager 2 spacecraft by Limaye and Sromovsky (1991). Sromovsky et al. (1993) used these Voyager data, binned at 1 degree intervals in latitude, to establish the "smooth Voyager wind profile". Wind speeds derived for individual features on HST images taken in subsequent years showed no significant deviations from this profile, except for features which resemble bright companion clouds to structures such as Dark Spots (Hammel and Lockwood 1997; Sromovsky et al. 2001b, 2001c). Sromovsky et al. (2001c), however, suggest that the zonal wind profile as derived from HST data in 1998 may show slight changes from the smooth Voyager profile.

The first wind speed experiments at high spatial resolution tracking small-scale features were conducted using Voyager 2 data. Smith et al. (1989) noted a large variation of wind speeds per zonal bin on Neptune, particularly at the latitudes of the Great Dark Spot (GDS) and Dark Spot 2 (DS2). This was confirmed by Limaye and Sromovsky (1991), who investigated whether measurement errors might be the cause of the large dispersion in wind speeds. After a detailed error analysis, they found that the observed dispersion exceeded the variance that was expected, even when including only the most accurately measured cloud features in the same latitude bin. They thus concluded that the large dispersion in wind speeds is most likely a real characteristic of the atmosphere of Neptune.

We note that in both the Voyager and HST experiments the sampling time of images is tens of minutes or more. When wind speeds are extracted from the motion of small cloud features, one has to be confident to 'track' the same feature from image to image. This can only be done by imaging Neptune with a high cadence, i.e., when the sampling interval is small-minutes rather than hours/days, since cloud features tend to change morphology on minute time-scales. Limaye and Sromovsky (1991) reported that in their study of clouds in the 1989 Voyager images of the several hundred cloud features they measured, they could only track reliably the positions of a few for more than 8 hours (half a Neptune rotation). With this in mind, we observed Neptune with the Keck adaptive optics (AO) system over 2 consecutive nights, during the full 4 hours each night that Neptune was observable. Unfortunately, a 4-hr baseline is too short to accurately measure the mean zonal flow, as such measurements are most accurate when features can be observed over several rotations (e.g., Sromovsky et al. 2009). The value of our data, however, is that with an integration time of only 60 seconds the 4-hr timeslots enabled us to build up a data base with enough images to derive relative velocities in Neptune's atmosphere for a total of almost 200 cloud features. We note that, although we mention velocities throughout the paper, we show the results in units of rotation period of features.

Using this data base, we address the following questions in this paper: (1) When the error in feature identification is minimized, is there a large variation in relative speeds per zonal bin, i.e, during a single 4-hour observation are there large variations in the east-west velocity at effectively the same latitude? (2) Is the observed variation significantly different from that expected from measurement errors? (3) Do the cloud motions give any indication of the cause of the variation? (4) Are large-scale features coherent during observations or do they tend to shear apart? We complemented our data base with service observing images taken 9 days later to address the latter question.

\section{Observations}

\subsection{Data acquisition and reduction}

On August 20, 21 and September 1, 2001 UT, we imaged Neptune in $\mathrm{H}$ band (1.4-1.8 micron) using the slitviewing camera (SCAM) of the NIRSPEC instrument, coupled to the Adaptive Optics (AO) system (Wizinowich et al. 2000a, 2000b; Johansson et al. 2000) at the W.M. Keck II telescope in Mauna Kea, Hawaii. SCAM contains a PICNIC $\mathrm{HgCdTe}$ chip with $256 \times 256$ pixels. The plate-scale is $0.0167 \pm 0.0002$ arcseconds and was calibrated using binary stars of known separation as documented in the HIPARCOS catalogue (Roe 2002). The field of view, at $4.3^{\prime \prime}$, is slightly larger than Neptune, which had an angular size of 2.34" at the time of our observations. Angular resolution was determined by measuring the full width at half maximum (FWHM) of the point-spread function (PSF) of stars slightly fainter than and within 5 degrees of Neptune. The angular resolution was $0.05^{\prime \prime}$, which corresponds to $\sim 1350 \mathrm{~km}$ at the center of Neptune's disk. We note that the actual angular resolution on Neptune may be slightly worse $\left(\sim 0.06-0.07^{\prime \prime}\right)$, as Neptune itself was used for wavefront sensing, and the AO system at that time had not yet been optimized to observe objects with a large angular size (see e.g., de Pater et al. 2002, 2005; van Dam et al. 2004).

We took a total of 58 images on August 20, 76 images on August 21, and 16 images on September 1. Observations spanned approximately 4 hours $(\sim 6: 20$ to $10: 20 \mathrm{UT})$ on August 20 and 21, and 50 minutes (9:20 to 10:10 UT) on September 1. The integration time for each image was 60 seconds, which provided enough signal-to-noise, did not 
saturate the detector, and assured that feature smearing was small compared to one resolution element. (A 60-second exposure corresponds to smearing of 0.37 degrees of longitude, or $\sim 160 \mathrm{~km}$ at disk center for features with drift rates equal to the 16.11 hour internal rotation rate of Neptune.) Short integration times also enabled a dense sampling of images over time, minimizing potential misidentifications of features due to changes in morphology. We typically took sequences of 5 images, with small dithers in between. Image sequences were interrupted to take PSFs, flux and plate scale calibrators. The maximum gap between Neptune images was 32 minutes on August 20 and 50 minutes on $\mathrm{Au}-$ gust 21 .

We reduced images using standard infrared data reduction techniques of sky subtraction, flat fielding and medianvalue masking to remove bad pixels.

\subsection{Imaging results}

Figure 1 shows images taken near the beginning and end of each night on August 20 and 21, and one typical image from September 1. Figure 2 shows cylindrical map projections. We used the many cloud features in these images as tracers of Neptune's wind profile, as discussed in Sect. 3. In this section, we describe the appearance of the images, including cloud locations.

The brightest clouds typically appear at mid-latitudes and form thin circumferential bands of clouds as described before by Max et al. (2003). These bands typically do not span all longitudes and do not precisely follow lines of constant latitude. At northern mid latitudes (NML) there is a single band of clouds centered at a planetocentric latitude of $+27^{\circ}$. A bright cloud occasionally appears at $+35^{\circ}$ and there are no cloud features between $+35^{\circ}$ and $+60^{\circ}$. Due to the tilt of Neptune's North Pole away from Earth, latitudes north of $+60^{\circ}$ are not visible.

In contrast to the single cloud band at NML, the southern mid latitudes (SML) show 3-5 bands of clouds at $-22^{\circ}$, $-30^{\circ},-35^{\circ},-44^{\circ}$ and $-48^{\circ}$. As in Max et al's (2003) images, this region contains the brightest clouds, and is therefore the most eye-catching area on Neptune's disk. The brightest clouds on the images are visible on the Eastern limb (right limb on the planet) on August 20 and on the Western limb on August 21. These brightest features are separated by 2 planet rotations and are likely the same feature. Max et al. (2003) posed the question whether these regions
Fig. 1 H-band (1.4-1.8 $\mu \mathrm{m})$ images of Neptune taken at 7:15 and 10:24 UT on August 20, 2001 (panels a and b), 6:20 and 10:21 UT on August 21, 2001 (panels $\mathbf{c}$ and d) and September 1, 2001 (panel e) using the "slit-viewing" camera SCAM of NIRSPEC coupled to the AO system. The dark stripe is the spectroscopy slit, which blocks out light to the camera. The reference grid (panel f) shows lines at 20 degrees separation in both longitude and latitude

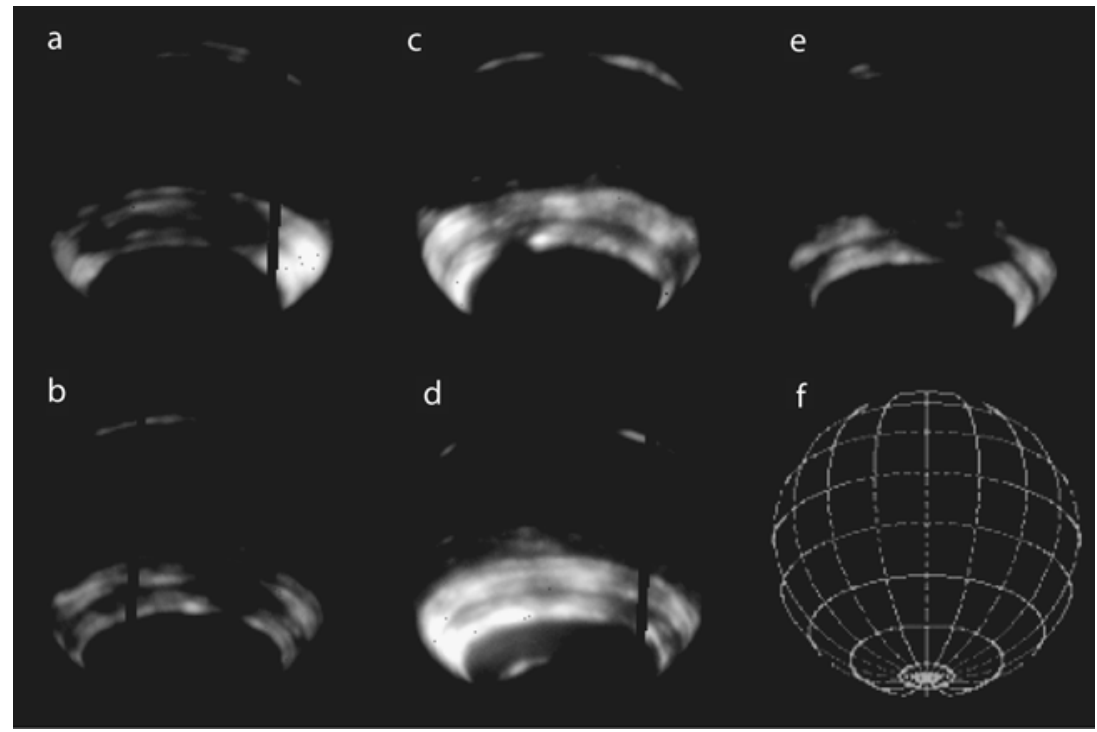

Fig. 2 Longitude-latitude map projections of the Neptune images in Fig. 1. By combining the images from August 20 and 21 , the total map grid spans 360 degrees in longitude

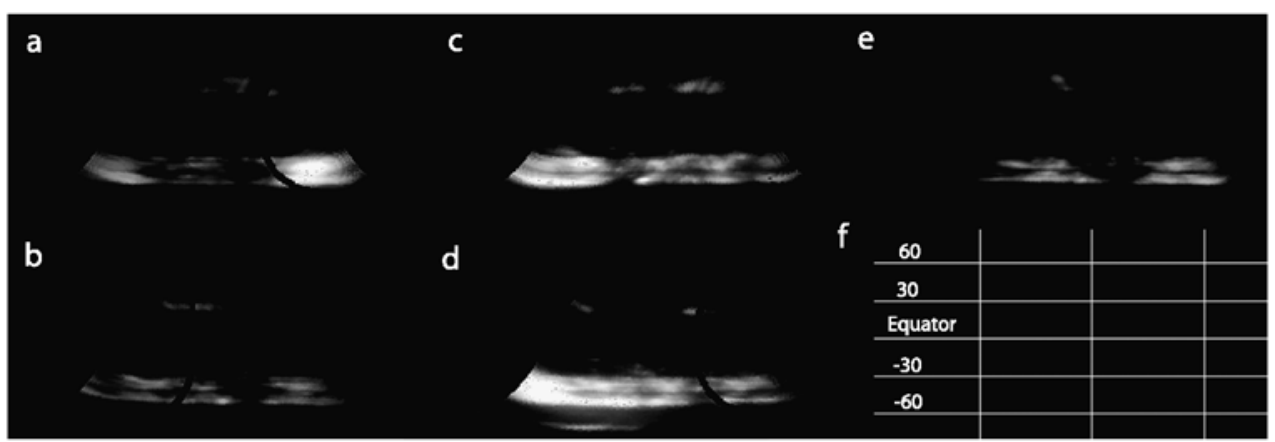


Fig. 3 Orthographic map projections centered on the South Pole of the images in Fig. 1. Latitude circles on the reference grid are at 30-degree separations. A faint cloud feature near a latitude of $70^{\circ} \mathrm{S}$ on 20 August is indicated with an arrow

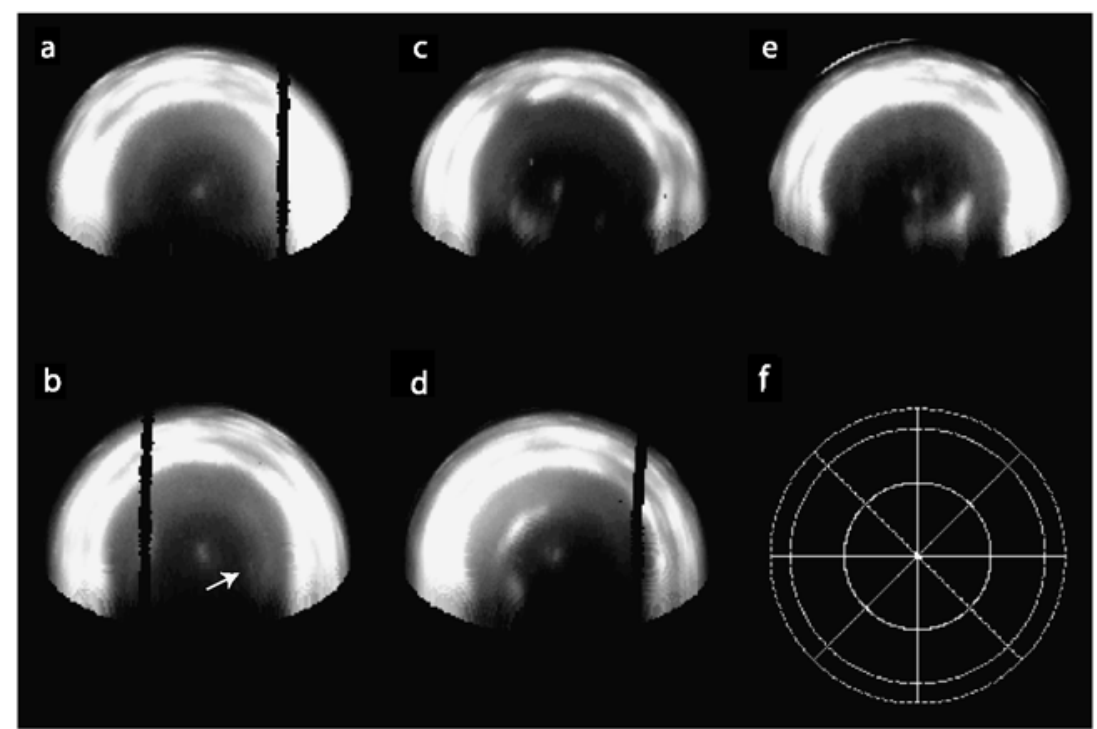

are coherent long-lasting features, or whether they disperse, as one might expect for clouds at different latitudes. Unless they are coherent objects, coherent features may result from vortices, standing waves, stagnation points, or places of upwelling. The series of images taken on August 21 show that the areas are bright in part because of the foreshortening effect near the limb. This suggests that small bright clouds that happen to line up near the limb may cause the bright regions. Moreover, the clouds must be somewhat transparent, i.e., not optically thick, to cause this effect.

Clouds at latitudes surrounding the equator are faint and have a more compact appearance than clouds at midlatitudes. The Equatorial Latitudes (EL) extend approximately from $-30^{\circ}$ to $+30^{\circ}$. During these observations there were many faint cloud features just north of the equator, a region that is often cloud-free. When we started the observations on August 20, there were features on the eastern limb, but these soon rotated out of view. Diffuse clouds in this area span $\sim 80-85^{\circ}$ of longitude west of the central meridian at the end of the night on August 21. The features are truly ephemeral, as they appear to develop and dissipate on minute timescales.

On September 1 several bright clouds are visible, reminiscent of a dark spot associated with "companion" clouds (sometimes referred to as the "northern cloud complex") discussed by Stratman et al. (2001) and Sromovsky et al. (2002). Latitudes just south of the equator between $-15^{\circ}$ and $0^{\circ}$ appear to be cloud free.

To best show features near Neptune's south pole, we show a (south) polar map projection in Fig. 3, with a contrast that emphasizes faint cloud features. This figure reveals that there is a very faint single faint cloud feature near the South Pole between -67 and $-70^{\circ}$ latitude on August 20 . The next day (August 21), at the same latitude, there is a string of 4 bright clouds that span $115^{\circ}$ of longitude. At the beginning of the observing session, these clouds are just visible at the "backside", but towards the end of the night the four features are quite prominent. They do not all fall on the same latitude circle. These features (or similar ones) are also visible on September 1. Clouds at this latitude were first identified by Hammel (1989); Smith et al. (1989) identified them on Voyager images, and referred to them as South Polar Features (SPFs). Sometimes there is a single feature, and at other times they appear to extend over a broad longitude range; Sromovsky et al. (1993) report that they reached a maximum extent of $170^{\circ}$ in longitude during the Voyager encounter. The SPFs have subsequently been seen in many HST and AO observations. These features are known to evolve rapidly, as also shown in our data set. It is interesting to note that the east longitude of this train of bright clouds coincides with the numerous faint clouds visible at equatorial latitudes on August 21 (Fig. 1). The region immediately surrounding the South Pole, -70 to $-90^{\circ}$ is distinctly dark (no obvious haze) with the exception of the bright dot at the South Pole. This dot (easiest to see in Fig. 3) is visible in all but 3 images and is used to verify image navigation.

\section{Data analysis}

To find zonal and meridional wind speeds, we assume that each cloud samples the local wind speed and that the altitude of each cloud feature above the 1-bar reference spheroid is constant. We used the following scheme to track the 2dimensional velocities of cloud features: (1) Create a map projection of each disk image; (2) Locate each cloud feature in a time series of maps to give latitude and longitude versus time for each; (3) Plot longitude versus time and latitude versus time for each feature and calculate the linear fit for each graph to determine rotational periods, zonal and meridional 
wind speeds. In the following sections we describe each step of the cloud-tracking scheme in detail.

\subsection{Map transformation and image navigation}

To facilitate feature identification, we transformed each disk image into a longitude-latitude map. The axes of the map correspond to degrees of planetocentric longitude and latitude. Figure 2 shows map projections of the disk images shown in Fig. 1. The map transformation rectifies the tilt of the planet indicated by the position angle and observer sub-latitude, then transforms from Cartesian image plane coordinates to planetocentric spherical coordinates. JPL's Horizons online ephemeris computation system (http://ssd.jpl.nasa.gov/horizons.html) provided ephemeris inputs to the transformation: North Pole position angle $\left(351.98^{\circ}\right)$, observer planetographic sub-latitude $\left(28.27^{\circ} \mathrm{S}\right)$, Neptune-Earth distance ( 29.15 A.U.), polar and equatorial radii $(24341 \mathrm{~km}$ and $24764 \mathrm{~km}$, respectively).

Figure $4 \mathrm{a}$ shows the map projection of a line grid. As you move away from the sub-observer point the thickness of the transformed line and thus the uncertainty increases greatly. To minimize errors due to viewing geometry, cloud features were not tracked near the limb, where the errorbars in Fig. 4 increase.

One of the more significant sources of error is the uncertainty in finding the center of the planet. Unfortunately, the position of the inner satellites, even though we could locate satellites on some of the images, are too inaccurate to help determine the center of Neptune (e.g., Dumas et al. 2002; Jacobsen and Owen 2004). We found the center of the planet by fitting the observed disk to an oblate planet with a geometry based on the JPL ephemeris. The limb was visualized using the Canny edge detection routine (Canny 1986). The cloud feature at the South Pole, assumed to be exactly at the south pole, was used to verify navigation, where its center was determined via a Gaussian fit. Although the dot may not be exactly at the South Pole (Luszcz-Cook et al. 2010), the planet center deduced from the South Pole dot agreed with the center deduced from limb fitting within 1 image pixel. To illustrate the effect of navigation errors on cloud position, Figs. $4 \mathrm{~b}$ and $4 \mathrm{c}$ show longitude-latitude grids that have been transformed from a disk image using a center that is slightly off. Figure $4 \mathrm{~b}$ shows the increase in error (indicated by the black and white pixels) due to a 1-pixel error in the center towards the east. In Fig. $4 c$ the black and white pixels similarly show an error in the center towards the north.

\subsection{Feature location and wind velocities}

As shown in Fig. 1, cloud features other than the South Pole dot are typically elongated in longitude and are often asymmetric with morphologies that evolve on minute time-scales. a

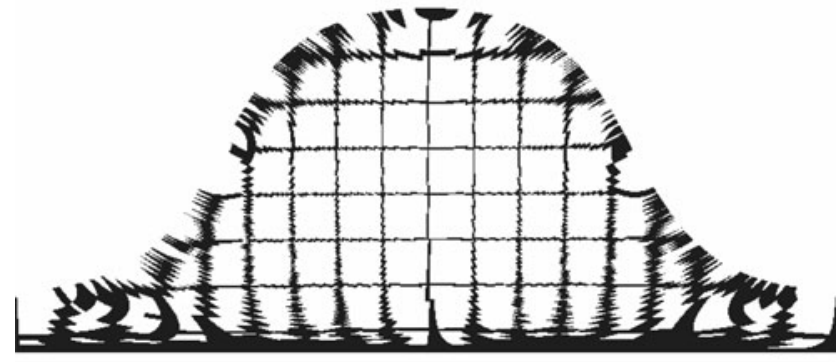

b

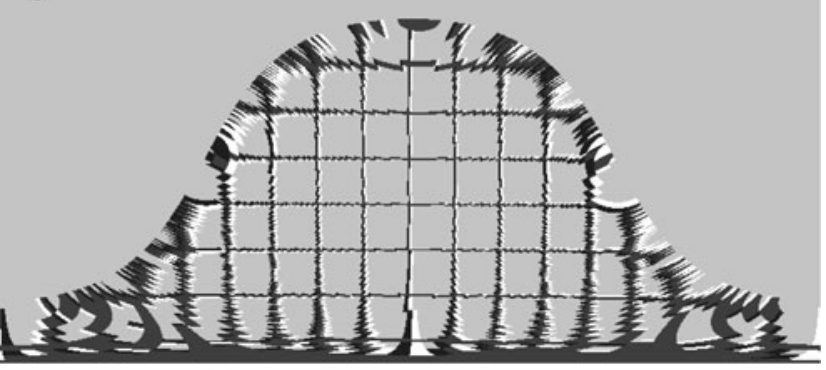

c

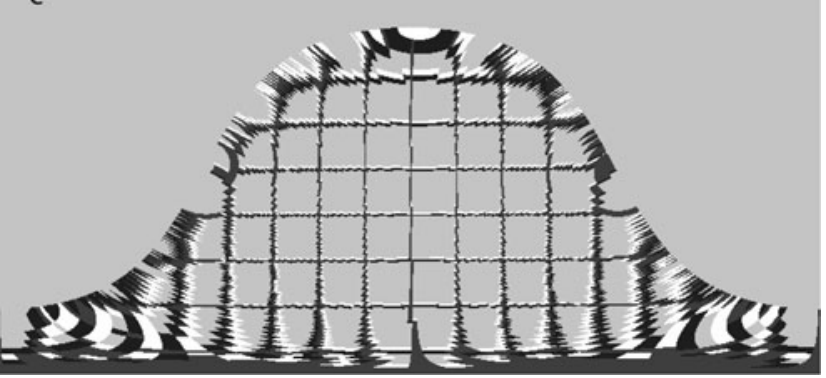

Fig. 4 Navigation errors for latitude and longitude. Constant latitude and longitude lines on a sphere are transformed with the center shifted in the direction (in planetary coordinates): (a) Without shifting, (b) 1 pixel to the East, (c) 1 pixel to the North. In $\mathbf{b}$ and $\mathbf{c}$, the transformed grid is subtracted from the unshifted grid, so white indicates grid points on the shifted grid that were not on the unshifted grid, black indicates grid points on the unshifted grid that were not on the shifted grid, grey indicates common points between the shifted and unshifted grids. Hence black and white in panels $\mathbf{b}$ and $\mathbf{c}$ show the errors due to a shift in the assumed center of the disk

Such features generally cannot be fit with Gaussians. Instead, we superposed contours at $60 \%, 70 \%$ and $80 \%$ of the maximum intensity on the features. The feature's location was defined as the midpoint of the longitudinal and latitudinal extent of the contours, and the difference in location using different contour levels was used as an uncertainty in feature location. Graphs of each feature's longitude as a function of time are shown in Fig. 5, with superposed linear least square fits for each feature. The errors indicated on each data point is the total uncertainty in the feature's location; i.e., the error in feature location was added in quadrature to the 1-pixel uncertainty in locating the center of Neptune's disk.

Each graph contains features in a particular latitude range (a few degrees in extent; see Sect. 2.2), as indicated by the 

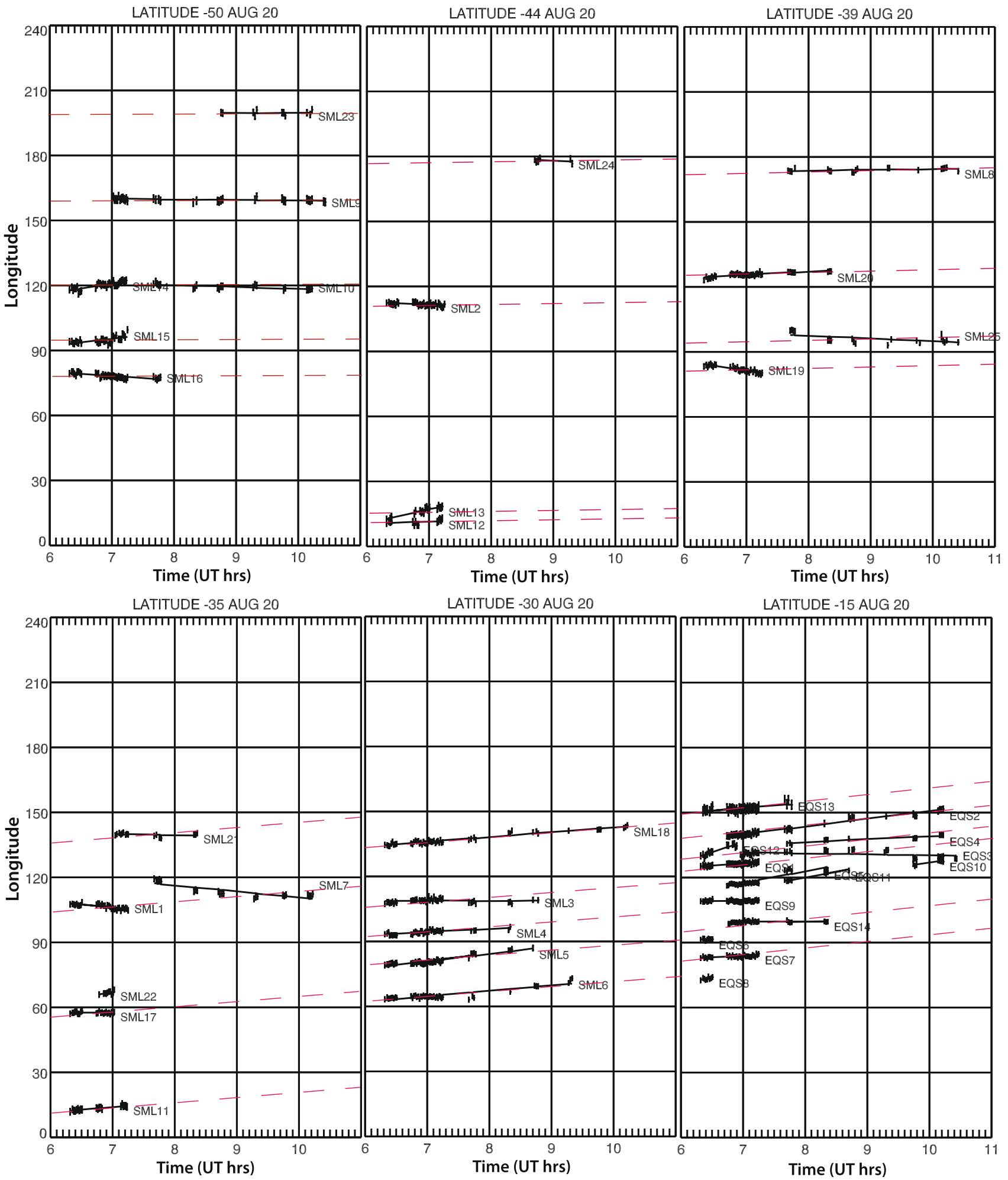

LATITUDE -30 AUG 20

LATITUDE -15 AUG 20

Fig. 5 Cloud tracking results for small features on August 20 and 21. Each graph shows West longitude versus time for cloud features in a particular latitude bin. The central latitude is indicated at the top of each plot. The latitude bins are a few degrees in latitude; they essentially correspond to the width of the narrow bright bands seen in Figs. 1 and 2. A linear fit to each set of data points is superposed. The zonal velocity of the feature can be derived from the slope of the lines. Different linear fit slopes indicate that clouds do not travel at the same zonal speed; a horizontal line indicates that the feature's velocity relative to the planet's rotation is zero. In addition, the superposed (red) dashed lines show the Voyager mean zonal wind velocity (relative to the planet's rotational period of $16.11 \mathrm{hrs}$ ) at the latitude of the features (from Fig. 8). Although these lines do go through many of the data points, there are significant differences between the Voyager derived velocities and many of the fits though our data points 

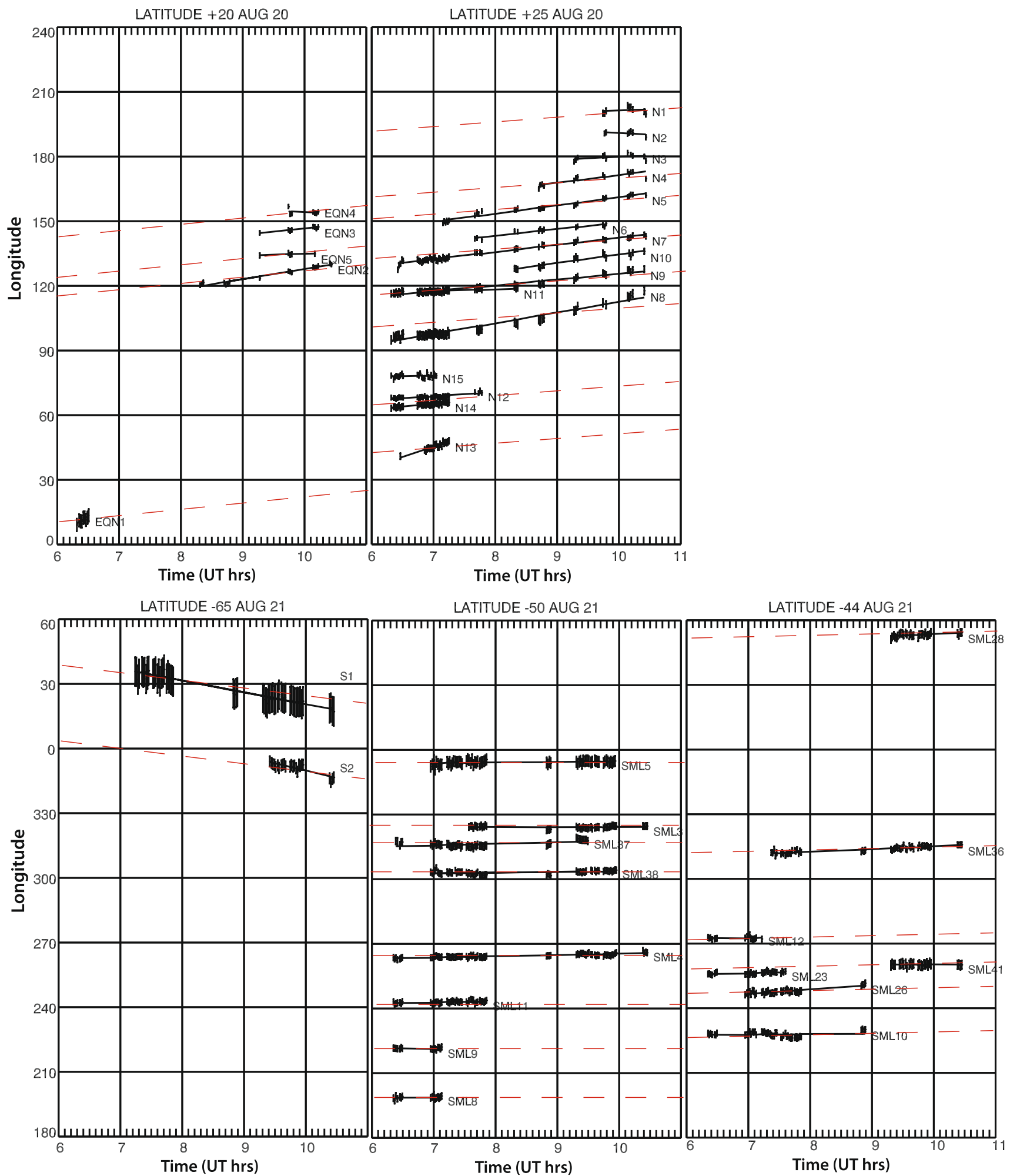

Fig. 5 (Continued)

central latitude at the top of each graph. Each feature is indicated by a unique label, e.g., SML for south mid-latitudes followed by a sequential number. The rotational period $P_{\text {rot }}$ and zonal velocity $V$ of the feature can be derived from the slope of the line, $\Delta$ (longitude $) / \Delta($ time $)$ :

$P_{\text {rot }}=360 /(360 / 16.11+\Delta($ longitude $) / \Delta($ time $))$ 


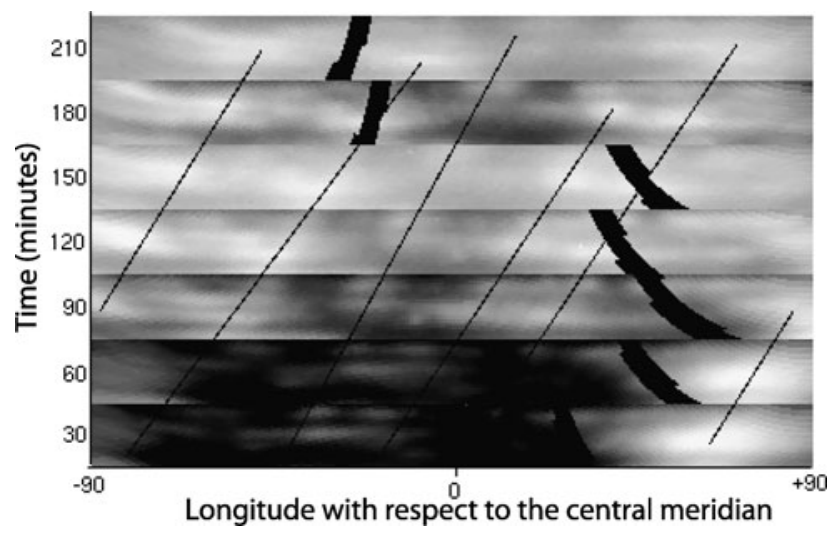

Fig. 6 Position versus time image strips. These strips illustrate changes in feature morphology, as well as the relative motion of features at the same latitude. Cloud images for a latitude range $20^{\circ}-50^{\circ} \mathrm{S}$ are spaced according to a 30-minute time interval. The lines graphically illustrate the motion of several features at the same latitude over a 4-hour time-span (these lines do not represent best fits to the data, but merely illustrate the effect seen in Fig. 5). Parallel lines indicate that corresponding features travel at the same speed. This plot shows that many features, even at the same latitude, exhibit different zonal velocities, and also shows that cloud features often survive less than 4 hours. Note that we used images separated by $1 \mathrm{~min}$ in time for the analysis of the data

$V=2 \pi R \cos \theta / P_{\text {rot }}$

with $R$ the planet's (equatorial) radius and $\theta$ the feature's latitude. The total error in the rotational period or zonal velocity follows from the rms deviation from a weighted linear least square fit.

Thus, features that move across Neptune's disk at the same speed as Neptune rotates around its axis (16.11 hrs), trace out a line like the horizontal grid lines on the graph. The velocities of features near a latitude of $\sim 50^{\circ} \mathrm{S}$ are very close to the planetary rotation rate. Most features at other latitudes deviate from this speed, as shown. In contrast to Limaye and Sromovsky (1991), none of the features showed a significant latitudinal motion. The former authors noticed an average northward speed of $25 \mathrm{~m} / \mathrm{s}$, which they suspect to result from a discrepancy between Neptune's actual rotation pole and the pole position as determined from spacecraft attitude determination. They note that most of this motion disappears if they would assume the small polar dot to indicate the position of Neptune's pole. Note that we assumed the polar dot to be at the planet's rotation pole throughout our analysis (Sect. 3.1). Sromovsky et al. (2001b) showed that long-lived cloud features also have no latitudinal motion. Only features that are tied to waves show some evidence of latitudinal motions. Notable exceptions, though, are the GDS, DS 2 and Scooter, which oscillate in latitude by $2^{\circ}-4^{\circ}$, with periods of order $1000 \mathrm{hrs}$ (Sromovsky 1991).

Figure 6 shows a time series of strips of longitude relative to the central meridian, centered at $35^{\circ} \mathrm{S}$ latitude and each covering $15^{\circ}$ of latitude. On this time series one can see the motion and evolution in morphology of features. To guide the eye, we have highlighted several features by superimposing straight lines to indicate their motion. Although one might expect the lines to be parallel for features at the same latitudes, the lines are clearly not parallel. This was our first clue that different features at the same latitudes moved at different speeds. Note that although we show here 6 strips for simplicity, we used all strips in our analysis.

\section{Discussion}

As discussed in Sect. 2.2, the concentration of clouds in circumferential bands at 30-50 degrees South is typical of AO images from Keck (Max et al. 2003; Gibbard et al. 2003; Luszcz-Cook et al. 2010) and CFHT (Roddier et al. 1998), and of 850 and $890 \mathrm{~nm}$ images from HST (Hammel and Lockwood 1997; Sromovsky et al. 2001b). These are likely similar to the bright cloud features seen in Voyager 2 images in methane absorption bands, and to those that appear as streaky white clouds at visible wavelengths (Smith et al. 1989; Limaye and Sromovsky 1991). However, what is striking is the apparent change in morphology: during the Voyager era, such prominent bands at southern midlatitudes had not been seen, as noted before by e.g. Hammel and Lockwood (1997). Moreover, Limaye and Sromovsky (1991) show that practically no cloud features were seen at $30^{\circ} \mathrm{S}$ in the Voyager images, and Sromovsky et al. (2002) point out that the bright band at $30^{\circ} \mathrm{N}$ became significantly more prominent between 1996 and 2002; our data show that this must have happened before 2001. In this paper we derive rotation periods for small cloud features in these infrared-bright bands and at the equatorial latitudes, i.e., in regions on the planet that have changed quite drastically since the Voyager era.

\subsection{Variation in zonal speeds}

In August 2001, Neptune was imaged over a period of 4 hours ( $\sim 1 / 4$ of the planet's rotation period) on each of two consecutive nights. Although the baseline for both nights combined is 28 hours, the typical rotation period of features (11-19 hours-Ingersoll et al. 1995) and the four-hour per night observing windows guarantee that on consecutive nights we usually see features on opposite 'sides' of the planet. Given these short baselines (4 hours), we do not attempt to derive an accurate mean zonal planetary wind profile, which would require data over at least 4 consecutive nights to get data on features over a long enough baseline to determine their rotation period (see e.g., Sromovsky et al. 2009). Rather, our goal is to take snapshots of the atmosphere to characterize the (distribution of) the dispersion in the rotational periods or wind speeds of cloud features. 
Fig. 7 (a) Planetocentric latitude versus rotation period for all cloud features tracked. The size of each plot symbol represents the time baseline for tracking. The largest symbol represents 3 to 4 hour baselines and the smallest symbols represent less than 1 hour baselines. The solid curve shows the smooth Voyager-derived wind profile from Sromovsky et al. (1993). Typical rms errors are $0.1-0.3 \mathrm{hr}$, with some up to 0.6-0.9 hr. The dashed lines show velocity contours for prograde winds; retrograde winds are shown by dot-dashed lines. The center dotted line shows the zero velocity line for the planet's $16.11 \mathrm{hr}$ rotation period. (b) Planetocentric latitude versus rotation period for all cloud features tracked. The size of the plot symbol represents the brightness of the feature tracked. The larger the plot symbol the brighter the feature. The curve is the smooth Voyager-derived wind profile based on Sromovsky et al. (1993). Note that the brightest features usually agree with the Voyager fit, with the notable exception of the feature near $50^{\circ} \mathrm{S}$
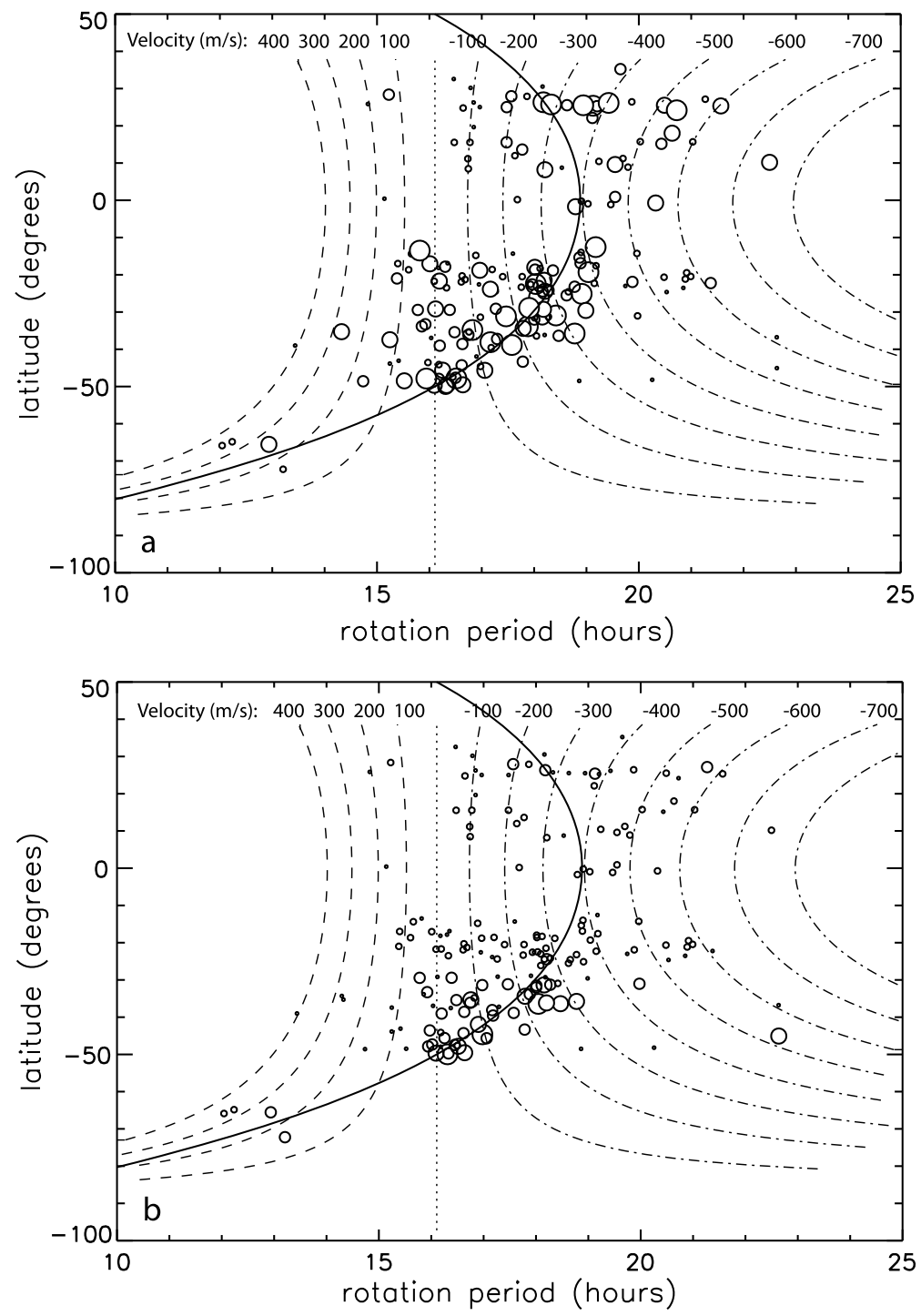

As discussed in Sect. 3.2, graphs of longitude versus time relative to the internal rotation of the planet are shown in Fig. 5, with superposed linear least square fits for each feature. Each plot contains the position versus time curves for features that are at a similar latitude, i.e., within a few degrees of the central latitude indicated at the top of each graph, or essentially within each narrow latitude band shown in Figs. 1 and 2. Thus the slope of the linear fit to each curve is proportional to the wind speed (or drift rate; note that the proportionality constant varies with latitude) and to the difference of the inverse of the rotation period derived for that particular feature (see (1) and (2)). The best-fit lines for features at $50^{\circ} \mathrm{S}$ are essentially parallel on both days, and hence features at $50^{\circ} \mathrm{S}$ latitude seem to travel at essentially the same speed, as one would expect for features that "move with the flow". At most other latitudes, however, not all lines are parallel, and non-parallel slopes suggest that either there are different velocities for different features or that not all features "move with the flow".
To demonstrate the scatter in velocity apparent in Fig. 5, the measured zonal drift rates are superimposed on Sromovsky et al's (1993) smooth Voyager Zonal Wind profile in Fig. 7. This figure demonstrates the large dispersion in rotation period, or wind speed, of the various features. In Fig. 7a the size of each symbol indicates the time interval over which features were tracked. The larger the symbol the longer the time interval. Typical rms errors in the rotation period vary from $0.1 \mathrm{hr}$ up to $0.3 \mathrm{hr}$ (though occasionally errors are as large as $0.6-0.9 \mathrm{hr}$ ) which is much smaller than the dispersion in the measurements. The uncertainty in the period depends on a combination of the individual measurement errors, indicated by the errorbars on Fig. 5, the baseline length and the linear least square fit through the data. Usually one expects the uncertainty in the period to be smallest for the longest baselines. Figure 7 a demonstrates that although the spread for features with short baselines may be largest, features observed over all time intervals show large 
variations in rotation period. In Fig. $7 \mathrm{~b}$ the size of the plot symbol represents the brightness of the cloud feature. The brightest features are usually correlated more tightly with the Voyager derived profile than faint features, regardless of baseline lengths, with the notable exception of one feature near $50^{\circ} \mathrm{S}$. We further note that some clouds at different latitudes, but similar longitudes, share the same wind speed. Sromovsky et al. (2001a, 2001b) report similar results. With the large differences in rotation period at any particular latitude, the spread in velocity is also very large; depending on latitude, a 4-5 hr difference in rotation period translates into variations of $\sim 500 \mathrm{~m} / \mathrm{s}$ ! Hence one naturally asks the question whether such variations can be real.

Data taken over the full $4 \mathrm{hr}$ baseline show gaps of 30$50 \mathrm{~min}$ (Sect. 2.1). With the variability in cloud features one may wonder whether the same feature was observed before and after the gap in time. In many cases features could be traced "over the time gap" by correlating neighboring features as well. In such cases the fit connects the data points. So we feel fairly confident that in most cases the same feature was traced over the entire time period, although disappearance, re-appearance and changes in morphology cannot ruled out.

On careful inspection of Fig. 5, one may notice that not all data follow the linear trends indicated by their least square fits. After the linear drift rate is subtracted from the data, some residuals appear to show oscillations, usually with a period similar to or exceeding the 4-hour baseline of our observations. Since features traversing the planet's disk in the same way reveal such oscillations with a different phase, the oscillations may be real; we discuss these in greater detail in Sect. 4.2. However, regardless of oscillations being real or not, such oscillations do affect the derived wind speed. For example, if features are tracked over a time period much less than the oscillation period, the linear fit through the data may be slightly off, which results in a misrepresentation of the wind velocities (while the rms error on this velocity may be small). Many of the baselines, however, are long enough to not be seriously affected by such oscillations, and there does not appear to be a difference in velocity dispersion between features with short and long baselines.

To further investigate the observed dispersion in rotational periods at particular latitudes, we superposed the expected Voyager derived mean velocity (from Fig. 7) on the graphs in Fig. 5. As the uncertainties in the data are relatively large, such lines do go through many of the data points, but certainly cannot fit the data for all features, and/or provide quite bad fits, which can be gleaned right away by looking at the slopes of the lines on the various plots-see, for example, lines at southern latitudes between $39^{\circ} \mathrm{S}$ and $15^{\circ} \mathrm{S}$ and all mid-northern latitudes. We thus conclude that there appears to be a large spread in rotation period, and hence zonal velocity, at many latitudes.
A large spread in velocities of features at the same latitudes was first noticed in measurements of small clouds in Voyager 2 data of Neptune (Smith et al. 1989; Limaye and Sromovsky 1991), in particular at the latitudes of the Great Dark Spot and Dark Spot 2. After careful inspection and error analyses of the Voyager data, Limaye and Sromovsky (1991) conclude that although measurement errors are significant at most latitudes, velocity dispersions over $25 \mathrm{~m} / \mathrm{s}$ cannot be attributed to measurement errors. In our data we find rms errors in the rotation periods of individual features of $0.1-0.3 \mathrm{hr}$, which corresponds to a typical rms velocity of order $25 \mathrm{~m} / \mathrm{s}$. (The precise numbers vary somewhat, depending on latitude.) Hence, in agreement with the previous study, we find a large dispersion in the east-west component of the velocity at several latitudes.

How can one explain such a large velocity dispersion? The first possible explanation that comes to mind is that the observed dispersion is due to the vertical shear of the zonal wind. Inversion of Voyager IRIS data suggests that at pressures between 0.030 mbar to $1 \mathrm{bar}$, the vertical wind shear over 1 scale height varies with latitude from $-20 \mathrm{~m} / \mathrm{s}$ to $40 \mathrm{~m} / \mathrm{s}$ (Conrath et al. 1989). However, the cloud features were detected at pressures between $\sim 0.02$ bar and $\sim 0.3$ bar (Gibbard et al. 2003), i.e., over no more than a few scale heights. Hence the maximum variation in the zonal velocity due to vertical shear and due to detecting features at very different altitudes is of order $50 \mathrm{~m} / \mathrm{s}$, certainly no more than $100 \mathrm{~m} / \mathrm{s}$, which is several factors less than our observed dispersion of the zonal velocity at fixed latitude. Therefore, we suggest that some of the observed variability is due to physical processes in the atmosphere, such as atmospheric waves.

The presence of atmospheric waves has been suggested in the past by several authors (e.g., Hammel and Lockwood 1997; Sromovsky et al. 2001a; Stratman et al. 2001). In the next section we make a first quantitative attempt to look for and identify such waves. In the last decade, it has become apparent that the Earth's oceans (Garrett and Munk 1979) and atmosphere (McLandress 1998), and numerical simulations (Sato et al. 1999) of rotating stratified fluids like Neptune's atmosphere are filled with Poincaré waves. These rotating stratified fluids not only allow for the existence of nearly dissipationless Poincaré waves, but also have been shown to be highly receptive (Herbert 1997; Barranco and Marcus 2005) to producing such waves (Benielli and Sommeria 1998). One possible excitation mechanism is tidal forcing by moons; we address this in some detail in the next section.

\subsection{Oscillations in longitude: possible wave behavior?}

Sromovsky (1991) reported longitudinal and latitudinal oscillations in the motions of the GDS and DS2. The periods of these motions are of order 800 hours for DS2 and 2550 
Fig. 8 Examples of oscillations displayed by features at southern mid-latitudes, on August 21. These graphs are obtained from Fig. 5 after subtraction of the linear fit as superposed on Fig. 5.

A polynomial fit is superposed on the data
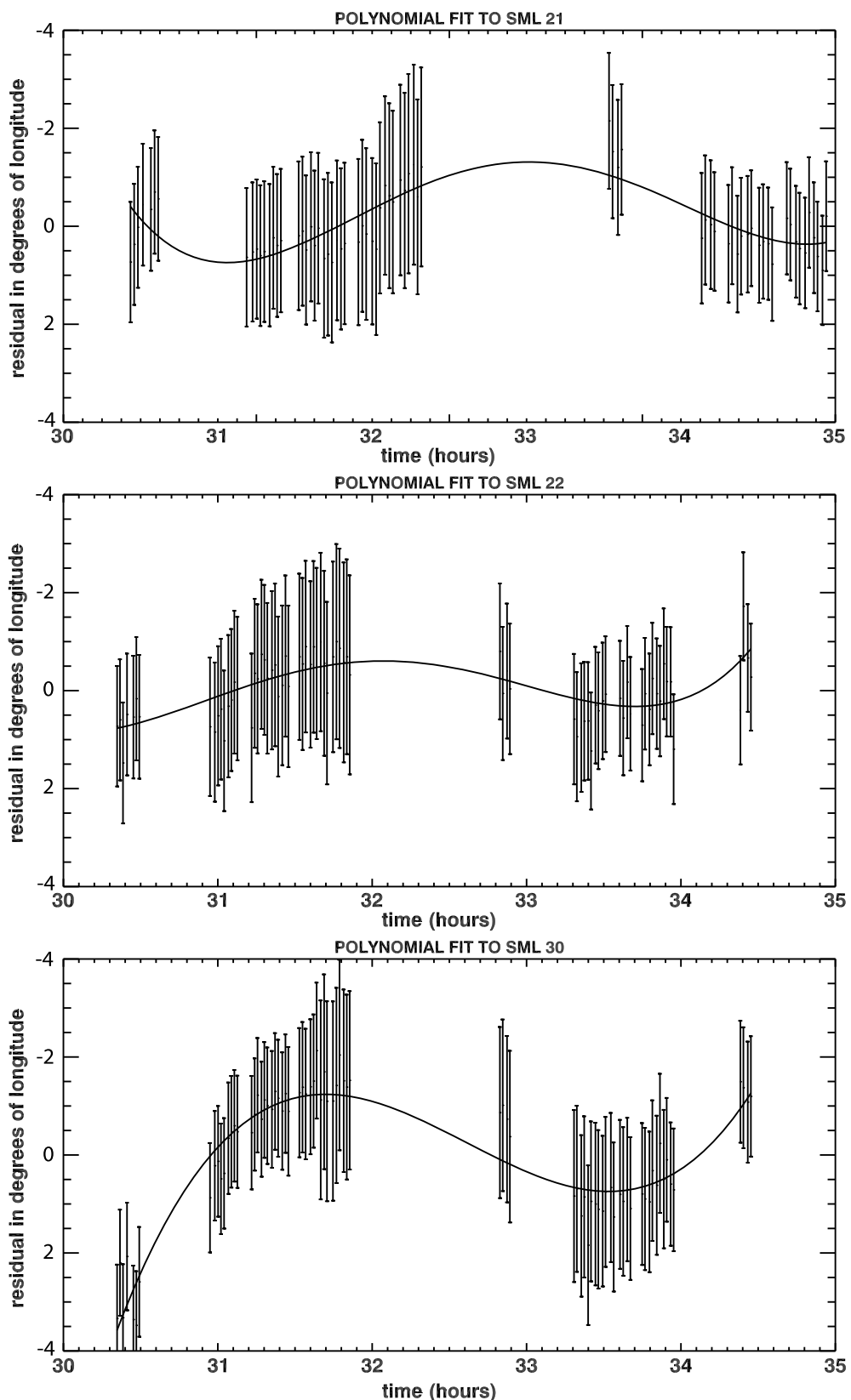

hours for the GDS. Our time coverage is much smaller, and hence we do not expect to see such oscillations in any feature. However, as mentioned in the previous section, when the slope is removed from the longitude versus time and latitude versus time graphs (in Fig. 5), the residuals of 17 cloud features at southern mid-latitudes (34, 36, 43,47 and $\left.49^{\circ} \mathrm{S}\right)$ suggest the presence of oscillations with typical periods of 4-8 hrs, i.e., of order half the planetary rotation period. The most convincing examples are shown in Fig. 8. The errors in the measurements as plotted are the total uncertainties in a feature's location, as described in Sect. 3.2. Polynomial fits are superposed on the data. We note that these features are at the same southern mid-latitudes that show a high dis- persion in local zonal wind-speeds as discussed in Sect. 4.1. The dominant frequencies of the residual displacements are $\sim 50-80 \mu \mathrm{Hz}$, which is much shorter than the Brunt-Väisälä frequency of $0.014 \mathrm{~Hz}$. Amplitudes range from $<1$ up to 5 degrees of longitude.

It is difficult to entirely convince oneself of the reality of the oscillations (see e.g., Fry and Sromovsky 2004). For example, the precise location of a feature could be influenced by a variety of effects: A single large feature might have components of time varying brightness that may translate into an effective longitude shift from the dimmer to the brighter component. Limb brightening of features approaching the limb may cause an apparent shift in a feature's lon- 
gitude. Navigation errors may play a role, as well as nonlinearity effects in the detector (i.e., residual pixel-to-pixel changes in the platescale).

However, we note that if the observed variations are real, the periods of the residual displacements and velocities (Fig. 8) are close to the M2 period of $7.24 \mathrm{hrs}$ due to the tidal forces from Triton. The M2 period is defined to be half of the orbital period of the moon as observed in the rotating frame of an observer on Neptune. On Earth the M2 waves are the largest amplitude Poincaré waves in the ocean, and they are responsible for the approximately twice-daily tides of the ocean.

Internal gravity waves are omnipresent in the convectively stable regions of most atmospheres (and oceans). They have a restoring force due to gravity, and, when they are not continuously forced, exist for frequencies (i.e., they have "natural" frequencies $\omega_{0}$ ) less than the Brunt-Väisälä frequency, $N$. For Neptune at the altitudes we sampled, $N=0.014 \mathrm{~Hz}$ (Fig. 8). Inertial waves are also omnipresent in most rotating systems. They have a restoring force due to the Coriolis effect, and, when they are not continuously forced, exist for frequencies less than $f$, the Coriolis parameter, which is $34.5 \sin (\theta) \mu \mathrm{Hz}$, where $\theta$ is the latitude. Poincaré waves are due to both gravity and Coriolis forces and exist for unforced waves for frequencies (or, equivalently, they have natural frequencies that lie) between $N$ and $f$. When any of these three types of waves are forced (most commonly by tidal forcing), the waves have the same frequency as the forcing frequency $\omega_{f}$. This is true even if this frequency is not in the allowable range of the unforced wave's natural frequencies-analogous to the response of a spring that is mechanically forced with an imposed frequency not equal to its own natural frequency. The amplitudes and phase shifts of the waves with respect to the forcing both depend on $\left(\omega_{f}^{2}-\omega_{0}^{2}\right)$ and on $\gamma$, which is the dissipation rate of the wave when it is unforced.

Observations of Poincaré waves on Neptune can be most easily detected if they create cloud displacements that are horizontal, rather than vertical in the atmosphere. The Poincaré waves of Neptune with fluid displacements that are nearly horizontal have frequencies that are close in value to the Coriolis parameter, which, in turn, is very close to the tidal forcing M2 frequency due to Triton. When waves are excited by tides (or any other mechanism) near their own natural frequencies, then the near-resonance causes them to have large amplitudes. Thus, M2 waves on Neptune should have both large amplitudes due to their near-resonance with M2, and be detectable because they produce near-horizontal displacements. (In contrast, Poincaré waves in which the displacements are vertical — which are hard to detect-have frequencies close to the Brunt-Väisälä frequency, which is a frequency far from M2.) To produce temporal oscillations in the east-west velocities of the tracked cloud features and no (or small) oscillations in the north-south velocities, the excited waves must have group velocities primarily in the east-west direction (and wave vectors and phase velocities primarily in the north-south direction).

The limited time series and large uncertainties of the data in Fig. 8 are not conclusive, but they are suggestive of M2 Poincaré waves due to Triton, in particular because this behavior was seen for 17 different features. The tidal force due to Triton acting on Neptune is approximately $1 / 3$ as large as the tidal forcing due to our Moon acting on the Earth, where our Moon excites an M2 response in the oceans (Lighthill 1978; Gill 1982) and upper atmosphere (Hines 1974; Ni et al. 2007). The moon with the next largest tidal force on Neptune after Triton is Proteus, but its tidal effect is less than one tenth of that of Triton, so we doubt that the oscillations in Fig. 8, if due to tidal effects, can be caused by any Moon other than Triton.

The fact that the temporal oscillations, as observed, are restricted to the east-west velocities of the tracked clouds rules out many other possible causes of the oscillations. For example, another way to create temporal oscillations is if a vortex were drifting with respect to one of our tracked cloud features. Each close encounter between a vortex and a cloud would induce large north-south velocities on the cloud, regardless of the direction in which the vortex approached the cloud, and therefore produce large temporal oscillations in the north-south velocity of the tracked cloud feature. Large temporal oscillations of the north-south velocities of the tracked features were not observed in our data.

Another way in which vortices could produce temporal oscillations in the locations of our tracked clouds is if the tracked clouds are associated with the vortices but not centered on them. Oscillations would be observed if the tracked clouds were off-center and circulated around the vortex centers. However, that arrangement would also produce large temporal oscillations in the north-south velocity of the tracked cloud features.

\subsection{Turbulence}

Turbulence is almost certainly present in the atmosphere of Neptune and creates velocity dispersions, but, one would expect turbulence to produce variations in both the north-south and the east-west components of the cloud velocities. Moreover, we would expect the temporal signature of the turbulence to be "noise" and not contain only a few temporal frequencies.

Can turbulence account for the variation in the zonal velocities in Fig. 5? Although we have shown that Sromovsky et al's (1993) smooth Voyager Zonal Wind profile can fit much of the data in Fig. 5, we have also shown that some of the measured rotation periods, or velocities, of the tracked clouds have error bars that are sufficiently small that they are 
true outliers and do not lie on the smooth curve. (A similar conclusion was reached by Limaye and Sromovsky 1991.) If the velocities of the outlying tracked clouds are true fluid velocities (i.e., if all of the tracked clouds move with the flow), then at some latitudes our results show that the zonal velocity varies by more than $400 \mathrm{~m} / \mathrm{s}$ over east-west distances of $\sim 20,000 \mathrm{~km}$. Because the flow is sub-sonic, the fluid should be nearly incompressible and therefore the velocity field should be divergence-free. To be divergence-free and have variations of the east-west velocity of order 400 $\mathrm{m} / \mathrm{s}$ would require large east-west gradients in the northsouth velocities of the tracked clouds (that were not observed by us or by Limaye and Sromovsky) or large vertical motions. Although vertical plumes cannot be ruled out by direct observations, the needed magnitudes to make the flow divergence-free are incompatible with the atmosphere's gradient Richardson number $\mathrm{Ri} \equiv N / \sigma^{2}$ number (the ratio of the potential energy that must be overcome by a vertically moving plume in a stratified atmosphere to the kinetic energy stored in the vertical shear of the horizontal flow) based on the values of the Brunt-Väisälä frequency $N$ and vertical shear $\sigma$ of the zonal flow reported above. We therefore conclude that the outliers observed by us and also the outliers observed by Limaye and Sromovsky (1991) are due to tracked clouds that do not move with the flow. The latter authors noticed rapid changes in the clouds, which they attributed to be caused by transient clouds on Neptune that decay and form rapidly due to condensation and sublimation resulting from weak vertical motions in the atmosphere. Our data support their conclusion, as we saw cloud features change morphology, and disappear/appear on timescales of minutes, in particular at the equatorial latitudes just north of the equator.

\section{Summary}

We present Adaptive Optics observations at near-infrared wavelengths, taken with the 10-m W.M. Keck I telescope on 3 different nights in August-September 2001 (UT 8/20, $8 / 21,9 / 1)$. Several tens of images were taken each night, which were used to investigate both the overall spatial brightness distribution, and potential short-term time variability on Neptune.

Our main conclusions regarding the spatial brightness distribution of cloud features can be summarized as follows:

(1) The spatial brightness distribution changed considerably between 1989 (Voyager era) and 2001. In particular, the eye-catching bright bands at southern midlatitudes, and the many bands in the north, had not been seen during the Voyager era.
(2) As also noted by e.g., Limaye and Sromovsky (1991), several latitude bands are cloud-free, such as the $\sim 70$ $90^{\circ}$ latitude band around the south pole, latitudes just south of the equator (between $-15^{\circ}$ and $0^{\circ}$ ), and between $+35^{\circ}$ and $+60^{\circ}$.

(3) The brightest features at southern latitudes extend latitudinally over several degrees and encompass several bright latitude bands. The bright regions observed by us appear on the limb of the planet, and may result from foreshortening effects rather than being true "coherent" features. This answers our question 4 posed in Sect. 1; although we cannot rule out that bright regions at other times could be "coherent" structures, tied together via vortices.

Wind velocities were derived by tracking small cloud features. Our main conclusions can be summarized as follows:

(4) Using different techniques, different data and a different wavelength range than Limaye and Sromovsky (1991), we tracked cloud features and found a large dispersion in the zonal velocities.

(5) Like Limaye and Sromovsky, we found about 2 dozen true outliers $(\sim 10-15 \%$ of the features tracked) whose velocities and uncertainties are very different from the Voyager mean wind zonal wind profile.

(6) We conclude that the outliers represent clouds that do not move with the local mass flow (otherwise, the velocity is not divergence-free).

(7) Temporal oscillations with periods of $\sim 4-8$ hours appear in 17 (about 10\%) of the cloud features that we tracked. Periods near 8 hours are close to being in resonance with the M2 forcing of Triton. This wave creates horizontal displacements that have a plausible chance of being detected.

As shown in this paper and by Limaye and Sromovsky (1991), it is clear that there are large velocity dispersions in Neptune's atmosphere. In addition, our data are suggestive of temporal oscillations in the east-west velocities of individual cloud features. To further investigate both the oscillations and velocity dispersions, we recommend to plan future observations at different phases of Triton (e.g., full, half, new), and investigate both the zonal and meridional velocities of cloud features (to date no reliable north-south velocities have been detected). Correlations between these two velocity components differ for waves, for random turbulence, and for small coherent vortices embedded in the zonal flow, and these differences could be exploited in future analyses to help determine the source of the variability.

Acknowledgements We thank Bruce Macintosh, Seran Gibbard, Henry Roe and Claire Max for their help during the early phases of this work. This research has been supported in part by the National Science Foundation Science and Technology Center for Adaptive Optics, managed by the University of California at Santa Cruz under cooperative 
agreement No. AST 9876783, as well as by NSF Grant AST-0908575 to the University of California. The data presented here were obtained at the W.M. Keck Observatory, which is operated as a scientific partnership among the California Institute of Technology, the University of California and the National Aeronautics and Space Administration. The Observatory was made possible by the generous financial support of the W.M. Keck Foundation. The authors extend special thanks to those of Hawaiian ancestry on whose sacred mountain we are privileged to be guests. Without their generous hospitality, none of the observations presented would have been possible.

Open Access This article is distributed under the terms of the Creative Commons Attribution Noncommercial License which permits any noncommercial use, distribution, and reproduction in any medium, provided the original author(s) and source are credited.

\section{References}

Asay-Davis, X.S., Marcus, P.S., Wong, M.H., de Pater, I.: Icarus 211, 1215 (2011)

Barranco, J.A., Marcus, P.S.: Astrophys. J. 623, 1157-1170 (2005)

Benielli, D., Sommeria, J.: J. Fluid Mech. 374, 117-144 (1998)

Canny, J.: IEEE Trans. Pattern Anal. Mach. Intell. 8, 679 (1986)

Conrath, B., Flasar, F.M., Hanel, R., Kunde, V., Maguire, W., Pearl, J., Pirraglia, J., Samuelson, R., Cruikshank, D., Horn, L.: Science 246, 1454 (1989)

de Pater, I., Gibbard, S.G., Macintosh, B., Roe, H.G., Gavel, D., Max, C.E.: Icarus 160, 359 (2002)

de Pater, I., Gibbard, S.G., Chiang, E., Hammel, H.B., Macintosh, B., Marchis, F., Martin, S., Roe, H.G., Showalter, M.: Icarus 174, 263 (2005)

Dumas, C., Terrile, R.J., Smith, B.A., Schneider, G.: Astrophys. J. 123, $1776(2002)$

Fry, P.M., Sromovsky, L.A.: Bull. Am. Astron. Soc. 36, 1103 (2004)

Garrett, C., Munk, W.: Annu. Rev. Fluid Mech. 11, 339 (1979)

Gibbard, S.G., de Pater, I., Roe, H.G., Martin, S., Macintosh, B.A., Max, C.E.: Icarus 166, 359-374 (2003)

Gill, A.E.: Atmosphere-Ocean Dynamics. International Geophysics Series, vol. 20. Academic Press, San Diego (1982)

Hammel, H.B.: Science 244, 1165-1167 (1989)

Hammel, H.B., Lockwood, G.W.: Icarus 129, 466 (1997)

Herbert, T.: Annu. Rev. Fluid Mech. 29, 245-283 (1997)

Hines, C.O.: Upper Atmosphere in Motion. Geophysical Monograph Series, vol. 18. American Geophysical Union, Washington (1974)
Ingersoll, A.P., Barnet, C.D., Beebe, R.F., Flasar, F.M., Hinson, D.P., Limaye, S.S., Sromovsky, L.A., Suomi, V.E.: Dynamic meteorology of Neptune. In: Cruikshank, D.P., Matthews, M.S. (eds.) Neptune and Triton. Univ. of Arizona Press, Tucson (1995)

Jacobsen, R.A., Owen, W.M.: Astron. J. 128, 1412 (2004)

Johansson, E.K., et al.: Proc. SPIE 4007, 600 (2000) and 20 colleagues

Lighthill, J.: Waves in Fluids. Cambridge University Press, Cambridge (1978)

Limaye, S.S.: NASA Spec. Publ. 494, 311 (1989)

Limaye, S.S., Sromovsky, L.A.: J. Geophys. Res. 96, 18941 (1991)

Luszcz-Cook, S.H., de Pater, I., Adamkovics, M., Hammel, H.B.: Icarus 208, 938 (2010)

Max, C.E., Macintosh, B.A., Gibbard, S.G., Gavel, D.T., Roe, H.G., de Pater, I., Ghez, A.M., Acton, D.S., Lai, O., Stomski, P., Wizinowich, P.L.: Astron. J. 125, 364 (2003)

McLandress, C.: J. Atmos. Sol.-Terr. Phys. 60, 1357 (1998)

Ni, X.J., Xiong, J.G., Wan, W.X., Ning, B.Q., Liu, L.B.: Earth Planets Space 59, 991 (2007)

Roddier, F., Roddier, C., Graves, J.E., Northcott, M.J., Owen, T.: Icarus 136, 168 (1998)

Roe, H.G.: Publ. Astron. Soc. Pac. 114, 450 (2002)

Sanchez-Lavega, A., Hueso, R., Perez-Hoyos, S.: Icarus 187, 510 (2007)

Sato, K., Kumakura, T., Takahashi, M.: J. Atmos. Sci. 56, 1005 (1999)

Smith, B.A., et al.: Science 246, 1422 (1989) and 64 colleagues

Sromovsky, L.A.: Science 254, 684 (1991)

Sromovsky, L.A., Limaye, S.S., Fry, P.M.: Icarus 105, 110 (1993)

Sromovsky, L.A., Fry, P.M., Baines, K.H., Limaye, S.S., Orton, G.S., Dowling, T.E.: Icarus 149, 416 (2001a)

Sromovsky, L.A., Frye, P.M., Dowling, T., Baines, K.H., Limaye, S.S.: Icarus 149, 459 (2001b)

Sromovsky, L.A., Frye, P.M., Dowling, T., Baines, K.H., Limaye, S.S.: Icarus 150, 244 (2001c)

Sromovsky, L.A., Frye, P.M., Baines, K.H.: Icarus 156, 16 (2002)

Sromovsky, L.A., Fry, P.M., Hammel, H.B., Ahue, A.W., de Pater, I., Rages, K.A., Showalter, M.R., van Dam, M.: Icarus 203, 265-286 (2009)

Stratman, P.W., Showman, A.P., Dowling, T.E., Sromovsky, L.A.: Icarus 151, 275 (2001)

van Dam, M.A., Le Mignant, D., Macintosh, B.A.: Performance of the Keck Observatory adaptive optics system. Appl. Opt. 43, 54585467 (2004)

Wizinowich, P., et al.: Publ. Astron. Soc. Pac. 112, 315 (2000a) and 17 colleagues

Wizinowich, P.L., Acton, D.S., Lai, O., Gathright, J., Lupton, W., Stomski, P.J.: Proc. SPIE 4007, 2-13 (2000b) 